\title{
A Case Study of S-Curve Regression Method to Project Control of Construction Management via T-S Fuzzy Model
}

Ting-Ya Hsieh

Professor, Department of Civil Engineering, National Central University, Chung-Li, Taiwan 320, R.O.C

Morris Hsiao-Lung Wang

Assistant Professor, Department of Civil Engineering, Vanung University, Chung-Li, Taiwan 320, R.O.C. Ph.D. candidate, Department of Civil Engineering, National Central University, Chung-Li, Taiwan 320, R.O.C., morris@msa.vnu.edu.tw

Cheng-Wu Chen

Ph.D. graduated, Department of Civil Engineering, National Central University, Chung-Li, Taiwan 320, R.O.C.

Follow this and additional works at: https://jmstt.ntou.edu.tw/journal

Part of the Civil and Environmental Engineering Commons

\section{Recommended Citation}

Hsieh, Ting-Ya; Wang, Morris Hsiao-Lung; and Chen, Cheng-Wu (2004) "A Case Study of S-Curve Regression Method to Project Control of Construction Management via T-S Fuzzy Model," Journal of Marine Science and Technology: Vol. 12: Iss. 3, Article 10.

DOI: $10.51400 / 2709-6998.2241$

Available at: https://jmstt.ntou.edu.tw/journal/vol12/iss3/10

This Research Article is brought to you for free and open access by Journal of Marine Science and Technology. It has been accepted for inclusion in Journal of Marine Science and Technology by an authorized editor of Journal of Marine Science and Technology. 


\section{A Case Study of S-Curve Regression Method to Project Control of Construction Management via T-S Fuzzy Model}

\section{Acknowledgements}

This paper is supported in part by the National Science Council of Taiwan, R.O.C. under projects No.- NSC 91-2626-E-238-003. 


\title{
Short Paper
}

\section{A CASE STUDY OF S-CURVE REGRESSION METHOD TO PROJECT CONTROL OF CONSTRUCTION MANAGEMENT VIA T-S FUZZY MODEL}

\author{
Ting-Ya Hsieh*, Morris Hsiao-Lung Wang**, and Cheng-Wu Chen***
}

Key words: fuzzy $S$-curve regression, $T-S$ fuzzy model, working capital management.

\begin{abstract}
In the contractual business, construction firms are generally more concerned with short-term financial strategies than the longterm ones. Working capital management is the central issue of all short-term financial concerns. Thus, it's urgent to study the cash portion of working capital management to rationalize the amount of cash and current assets possessed in certain time. The $S$-curve is quite suitable to represent the relationship between project duration and complete progress in practical usage of construction management. Based on the technique of Takagi-Sugeno $(T-S)$ fuzzy model, the fuzzy regression model is constructed for curve fitting problems. According to the cash flow of the example projects, this paper develops a practical S-curve regression model demonstrated and given tentative conclusions.
\end{abstract}

\section{INTRODUCTION}

The research of complex systems nowadays, such as engineering technology, environment and social economy, becomes so large in dimension and complex that the exact numerical data can not be obtained. Solving the problems caused by complex systems becomes very inefficient or even impossible if using the tradi-

Paper Submitted 10/03/03, Accepted 04/08/04. Author for Correspondence: Morris Hsiao-Lung Wang. E-mail: morris@msa.vnu.edu.tw.

*Professor, Department of Civil Engineering, National Central University, Chung-Li, Taiwan 320, R.O.C.

**Assistant Professor, Department of Civil Engineering, Vanung University, Chung-Li, Taiwan 320, R.O.C.

Ph.D. candidate, Department of Civil Engineering, National Central University, Chung-Li, Taiwan 320, R.O.C.

***Ph.D. graduated, Department of Civil Engineering, National Central University, Chung-Li, Taiwan 320, R.O.C. tional mathematical tools not constructed for dealing with high dimensionality models. Similarly, the traditional least square regression may not be applicable when dealing with curve fitting problems. In the past twenty years, some approaches containing fuzzy information have been noticed, as proposed in the literature $[2,6,7,9]$.

Tanaka et al. [9] developed a fuzzy linear regression model using linear programming techniques in 1982. In 1988, Diamond [2] resembled traditional least squares regression to establish fuzzy linear least squares models. Ruoning $[6,7]$ considered the rationality of metric definition, discussed the problem for least squares fitting of fuzzy-value data expressed as fuzzy numbers, and developed an $S$-shaped curve regression model for fitting this type of data.

However, being large-scaled, long duration, high cost, and complex-technical, the large public construction exists many uncertain factors. Because of these factors, to perform this kind of project is difficult, especially for the dispatch of working capital. In order to overcome the difficulties of controlling projects, the $S$-curves are widely used. They are valuable to project management in reporting current status and to predict the future of projects. Consequently, the $S$-type distribution is believed to be suitable in regression on construction management, social economy and so on. However, as far as we know, the fuzzy $S$-curve regression for large public constructions via Takagi-Sugeno fuzzy model remains an open area.

This study is discussed as follows. First, classic $S$ curve theory is recalled. Then, based on fuzzy set theory and fuzzy inference engine as well as center of gravity defuzzification, a $T-S$ type fuzzy $S$-curve is obtained for curve fitting problems. Finally, a numeri- 
cal example with simulations is given to demonstrate the methodology, and the conclusions are drawn.

\section{CLASSIC $S$-CURVE THEORY}

In biology and social economy, an $S$-shaped curve is often used to reflect the phenomena. It means that the trend of growth gets slow first and finally saturation rapidly. In practical problem of constructions, contractors' budgets are often performed on an overall basis. Changes in strategies and mix of contracts are very difficult to evaluate on such a basis [3]. Therefore, the principle of simulation with tools of computer was proposed to generate possible scenarios based on the specified strategies and the expected environment. The relationship between budgets and time limit for a project can be represented via $S$-curve fitting. A typical $S$-curve figure is shown in Fig. 1 . The $x$-axis and y-axis denote project duration and complete progress, respectively.

Miskawi [5] proposed an $S$-curve equation which can be used in a variety of applications related to project control. The $S$-curve model is of the following form:

$$
\begin{aligned}
P & =\frac{3^{T}}{2} \sin \left[\frac{\pi(1-T)}{2}\right] \sin (\pi T) \log \left(\frac{T+\left(1.5-T_{p}\right)}{T_{p}+T}\right) \\
& -2 T^{3}+3 T^{2}
\end{aligned}
$$

where $P$ denotes percentage completion of a project or an activity; $T$ denotes time at any point of the duration of a project or an activity; $T_{P}$ is shape factor.

Fig. 2 is plotted with various values of $T_{P}$ between $T=0$ and $T=100 \%$ duration and the envelope of curves for $T_{P}=5 \%$ and $T_{P}=95 \%$ in Eq. (1).

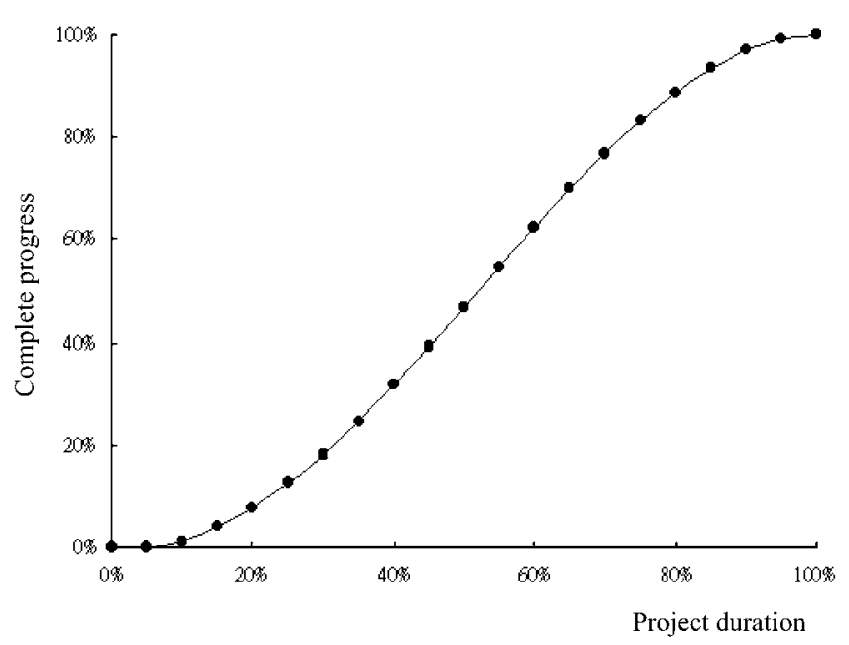

Fig. 1. Type $S$-curve figure.
Here we suppose we can exactly get all observed data taking part in the problems, but, actually, we may not know exact values, rather some approximation [7]. For this reason, the traditional fitting method may not be quite suitable. Before introducing fuzzy $S$-curve regression, we give some relative definitions and conclusions in the following.

\section{FUZZY SET THEORY}

Definition 1 [7]: Let $R$ is a real number set. A fuzzy set $\tilde{A}$ on $R$ is said to be a fuzzy number if the following conditions are satisfied:

(1) $\exists x_{0} \in R$, such that $\mu_{\widetilde{A}}\left(x_{0}\right)=1$; and membership function $\mu_{\tilde{A}}(x)$ is piecewise continuous; and

(2) $\forall \alpha \in(0,1], A_{\alpha} \equiv\left\{x \mid \mu_{\tilde{A}}(x) \geq \alpha, x \in R\right\}$ is a convex set on $R$,

where $x_{0}$ is the mean value of $\tilde{A}$ and $A_{\alpha}$ is a crisp set. The convex set means that $\forall x \in\left[x_{1}, x_{2}\right], \mu(\mathrm{x}) \geq \min (\mu$ $\left.\left(x_{1}\right), \mu\left(x_{2}\right)\right)$.

Definition 2: A fuzzy number $\tilde{A}$ is said to be bounded if $(\tilde{A}) \equiv\left\{x \mid \mu_{\tilde{A}}(x)>0\right\}$ is a bounded set, where $\operatorname{supp}(\tilde{A})$ is a crisp set.

Evidently for any $\forall \alpha \in(0, \alpha]$ the $\alpha$-level set, $\widetilde{A}_{\alpha}$, will be expressed as a closed interval $[p, q]$. Based on the fuzzy extension principle [12], linear operations about closed intervals are obtained as follows:

Lemma 1 [4]: Let $[a, b],[d, e]$ be closed intervals of real number. Then

$$
\begin{aligned}
{[a, b]+[d, e] } & =[a+d, b+e] ;[a, b]-[d, e] \\
& =[a-e, b-d],
\end{aligned}
$$

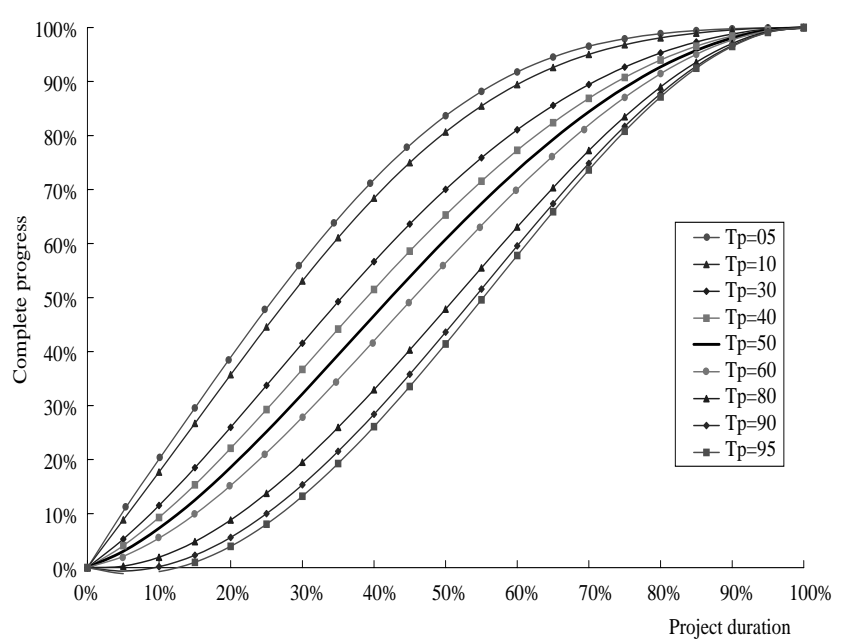

Fig. 2. Miskawi $S$-curve model. 


$$
\begin{aligned}
{[a, b] \cdot[d, e]=} & {[\min (d d, a e, b d, b e),} \\
& \max (a d, a e, b d, b e)] ; \\
{[a, b] /[d, e]=} & {[a, b] \cdot[1 / e, 1 / d] } \\
= & {[\min (a / d, a / e, b / d, b / e),} \\
& \max (a / d, a / e, b / d, b / e) .
\end{aligned}
$$

Remark 1: Given any operations which have commutative and associative characteristics, the operations of extension still have these characters.

From the theory of $\alpha$-level described above and decomposition theorem [4], we have

$$
\begin{aligned}
& (A * B)_{\alpha} \equiv A_{\alpha} * B_{\alpha} \\
& A * B \equiv \bigcup_{\alpha \in(0,1]}(A * B)_{\alpha}
\end{aligned}
$$

where * denotes any arithmetic operation; $A$ and $B$ are fuzzy numbers and $A * B$ will be a fuzzy number.

Remark 2: Wang and Chiu [11] proposed that the resultant fuzzy number is the same type as the original fuzzy numbers after the operation of addition, subtraction or multiplication. Namely, If $A$ and $B$ are the fuzzy numbers with the same type of membership function, then $A+B, A-B$ and $K \bullet A, K \in R$, are also the same type as $A$ and $B$.

Because the parameterizable membership function most commonly used in practice is triangular membership function, a useful concept described below is given. In which the membership function has three parameters and in general we assume that the peak of the membership function is 1 . Fig. 3 is an example of triangular fuzzy set to represent the fuzzy number with three crisp parameters.

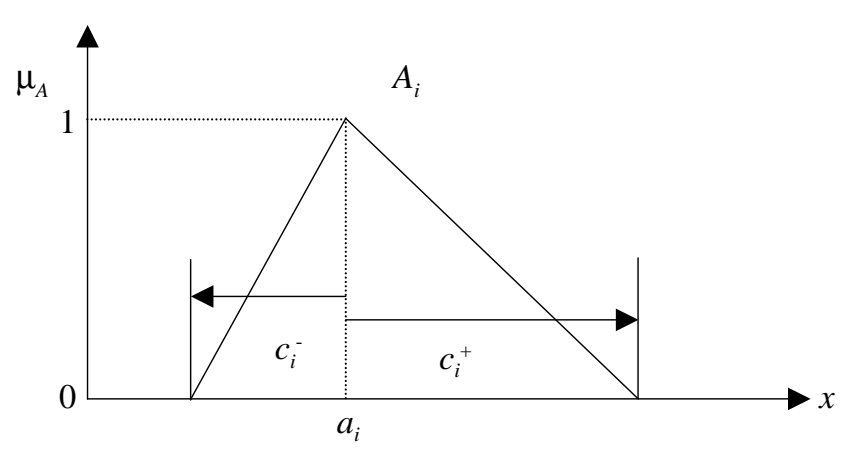

Fig. 3. Triangular fuzzy set.

\section{Definition 3:}

A fuzzy number $\tilde{A}$ is LR-type, if there exists positive constants $\beta>0, \gamma>0$ and

$$
\mu_{\widetilde{A}}(x)= \begin{cases}L\left(\frac{m-x}{\beta}\right) & \text { for } x \leq m \\ R\left(\frac{x-m}{\gamma}\right) & \text { for } x \geq m\end{cases}
$$

where $m$, a real number, is mean value of $\tilde{A} ; \beta, \gamma$ denote left spread and right spread, respectively and $L$ and $R$ are strictly nondecreasing continuous functions from $[0,1]$ to $[0,1]$ such that $L(0)=R(0)=1$ and $L(1)=R(1)=0$; moreover, $\tilde{A}$ could be represented as $(m, \beta, \gamma)_{L R}$. If $L=$ $R$ and $\beta=\gamma$, then the symmetric $L-L$ fuzzy number is denoted as $(m, \beta)_{L}$.

Lemma 2: Given two LR-type fuzzy numbers $\tilde{A}$ and $\tilde{B}$ of the same type, we have

$$
(m, \beta, \gamma)_{L R}+(n, \delta, \eta)_{L R}=(m+n, \beta+\delta, \gamma+\eta)_{L R},
$$

$$
(m, \beta, \gamma)_{L R}-(n, \delta, \eta)_{L R}=(m-n, \beta+\eta, \gamma+\delta)_{L R},
$$

if $\tilde{A}$ and $\widetilde{B}$ are not the same type, equations (8) and (9) would be inadequate.

In the next section, the concept of a so-called Takagi-Sugeno fuzzy model is utilized in fuzzy inference engine to establish a fuzzy $S$-curve regression model. Based on this regression model, an example in which the observed data are fuzzy numbers is given to demonstrate the proposed methodology.

\section{FUZZY $S$-CURVE VIA $T$ - $S$ FUZZY MODEL}

The $T-S$ fuzzy model was developed primarily from the pioneering work of Takagi and Sugeno [8], to represent the nonlinear relation of multiple input and output data, according to the format of fuzzy reasoning. Namely, the resulting overall fuzzy regression model, nonlinear in general, is achieved by fuzzy blending of each individual input-output realization (for more detail, please see [10]). Therefore, the ith rule of fuzzy inference is described by a set of fuzzy IF-THEN rules in the following form:

$$
\begin{aligned}
& R^{i} \text { : IF } x_{1} \text { is } \tilde{A}_{1}^{i}, y_{1} \text { is } \tilde{B}_{1}^{i} \text { and } \ldots \text { and } x_{n} \text { is } \tilde{A}_{n}^{i} \text { is } \tilde{B}_{n}^{i} \\
& \text { THEN } Y=a_{i k} x_{i}^{k}+b_{i k}
\end{aligned}
$$

where $n$ points $\left(x_{1}, y_{1}\right) \sim\left(x_{n}, y_{n}\right)$ and $\mathrm{k}$ order curve fitting is adopted, $R^{i}$ denotes the ith fuzzy inference rule and $r$ 
is the number of IF-THEN rules for $i=1,2, \ldots, r ; \tilde{A}_{p}^{i}$ and $\widetilde{B}_{p}^{i}(p=1,2, \ldots, n)$ are the LR-type fuzzy sets, and $x_{1} \sim x_{n}$ as well as $y_{1} \sim y_{n}$ are the premise variables. Using the center of gravity defuzzification, product inference, and single fuzzifier, the final output is inferred as follows:

$$
Y=\frac{\sum_{i=1}^{r} w_{i}\left[a_{a k} x_{i}^{k}+b_{i k}\right]}{\sum_{i=1}^{r} w_{i}}=\sum_{i=1}^{r} h_{i}\left(a_{i k} x_{i}^{k}+b_{i k}\right)
$$

with

$$
w_{i} \equiv \prod_{g=1}^{n}\left(A_{g}^{i}\left(x_{g}\right), B_{g}^{i}\left(y_{g}\right)\right), h_{i} \equiv w_{i} / \sum_{i=1}^{r} w_{i}
$$

in which $A_{g}^{i}\left(x_{g}\right)$ and $B_{g}^{i}\left(y_{g}\right)$ are the grade of membership of $x_{g}$ and $y_{g}$ in $A_{g}^{i}$ and $B_{g}^{i}$. In this paper, it is assumed that $w_{i} \geq 0, i=1,2, \ldots, r ; \sum_{i=1}^{r} w_{i}>0$. Therefore, $h_{i} \geq 0$ and $\sum_{i=1}^{r} h_{i}>1$.

Remark 3: The order $k$ of the consequence part can be determined by the method of added variable plots to find a suitable regression model (for more details, see [1]). In this paper, we only consider the factor of correlation coefficient. Therefore, the order $k$ is assigned hereafter to have a higher value of correlation coefficient.

\section{EXAMPLE}

To illustrate the procedure of this fuzzy regression

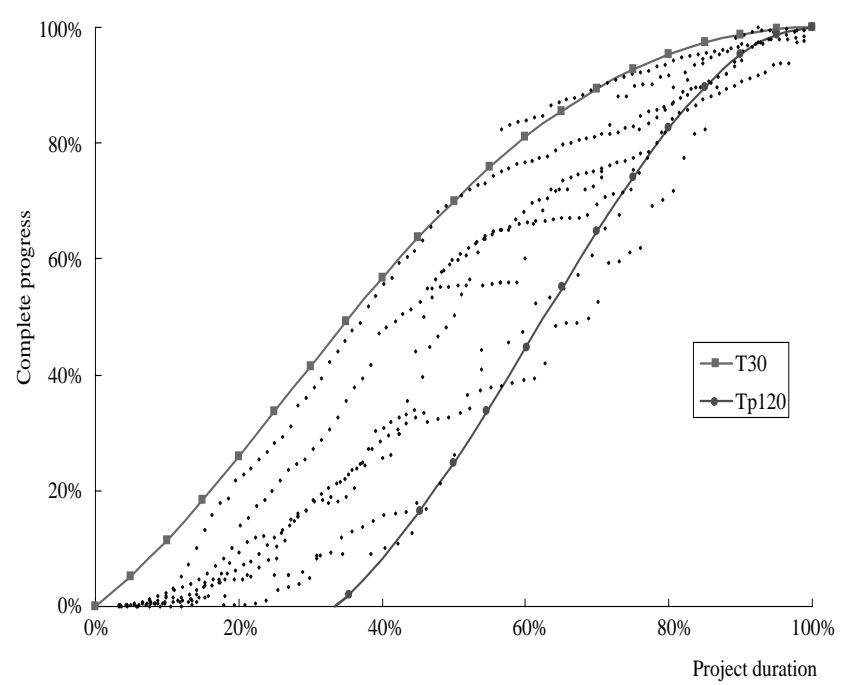

Fig. 4. The valuation data of six metro bids. model, refer to the following example project taken from Department of Rapid Transit Systems, Taipei City Government. The mean scale and duration of six metro bids data are 2.7 billions and 6 years or so. The data are normalized and transformed into the rate of percentage shown as Table 1. The first time of evaluation is $4.5 \%$ of total duration.

According to the data above, Figs. 3-5 will be discussed as follows. Fig. 3 shows the triangular fuzzy set to represent the fuzzy number with three crisp parameters. Hence, the expression $A_{i}=\left(a_{i}, c_{i}^{+}, c_{i}^{-}\right)$ stands for a triangular fuzzy number hereafter. In Table 1 , the data $X_{i}, Y_{i}$ are the model value $\tilde{X}, \tilde{Y}$ where $\tilde{X}=$ $\left(X_{i}, u_{i}, v_{i}\right)$ and $\tilde{Y}=\left(Y_{i}, r_{i}, s_{i}\right)$ are all triangular fuzzy numbers and $u_{i}=10 \%, v_{i}=10 \%, r_{i}=10 \%$, and $s_{i}=10 \%$ are the left and right spreads, respectively. Fig. 4 is the valuation data of six metro bids and Fig. 5 is plotted by the technique of the proposed fuzzy regression method in Eqs. (10-11). According to Fig. 5, the simulation shows the results: $y=-1293 x^{6}+4207 x^{5}-49.49 x^{4}+23$. $07 x^{3}-1.98 x^{2}+0.25 x$ [ $x$ denotes the completive percentage (\%), and $y$ is the time of duration (\%)] and it shows the square of the correlation coefficient, $R^{2}$ is 0.94 .

\section{CONCLUSIONS}

The least-squares method can usually be applied to the problems of curve fitting, but when the data are not obtained exactly, it may not be suitable. Therefore, we propose here an $S$-curve regression method for a better understanding of the issues involved. The aim is to develop a practical model for construction firms in Taiwan to rationalize the amount of cash and current assets possessed in certain time of duration.

Furthermore, Fig. 4 shows the data is under a

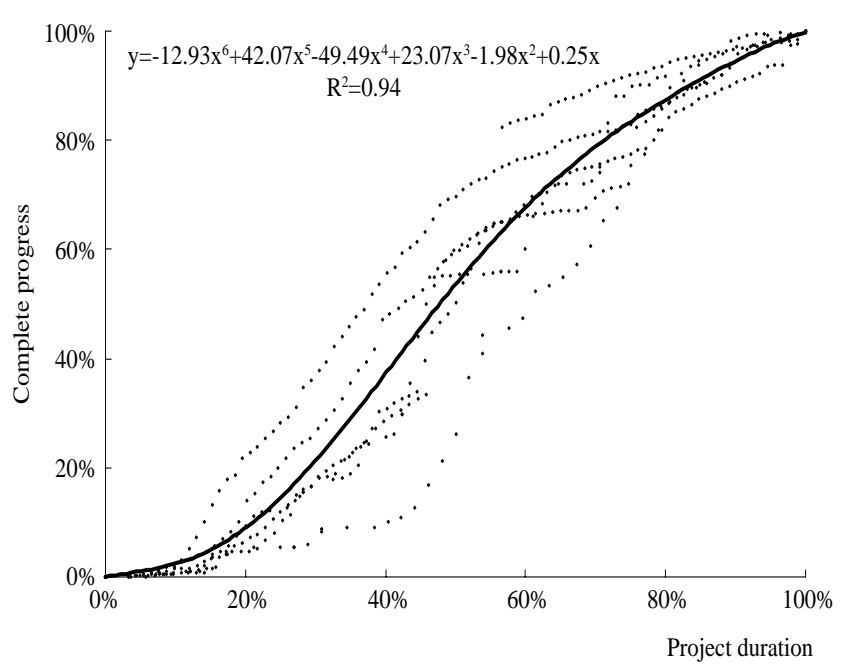

Fig. 5. An $S$-curve by fuzzy regression method. 
Table 1a. The observed data of the first metro bid

\begin{tabular}{|c|c|c|c|c|}
\hline $\begin{array}{l}\text { Valuation } \\
\text { times }\end{array}$ & $\begin{array}{l}\text { Valuation } \\
\text { month }\end{array}$ & $\begin{array}{l}\text { Accumulative } \\
\text { valuation total }\end{array}$ & $\mathrm{X} 1$ & Y1 \\
\hline To begin & $80 / 5$ & 0 & $0.00 \%$ & $0.00 \%$ \\
\hline 1 & $80 / 12$ & $74,144,882$ & $7.57 \%$ & $1.39 \%$ \\
\hline 2 & $81 / 1$ & $98,654,310$ & $8.73 \%$ & $1.85 \%$ \\
\hline 3 & $81 / 2$ & $127,656,501$ & $9.90 \%$ & $2.39 \%$ \\
\hline 4 & $81 / 3$ & $152,724,294$ & $10.98 \%$ & $2.86 \%$ \\
\hline 5 & $81 / 4$ & $178,169,197$ & $12.14 \%$ & $3.33 \%$ \\
\hline 6 & $81 / 5$ & $213,580,145$ & $13.27 \%$ & $4.00 \%$ \\
\hline 7 & $81 / 6$ & $255,800,965$ & $14.43 \%$ & $4.79 \%$ \\
\hline 8 & $81 / 7$ & $309,414,337$ & $15.55 \%$ & $5.79 \%$ \\
\hline 9 & $81 / 8$ & $378,460,468$ & $16.72 \%$ & $7.08 \%$ \\
\hline 10 & $81 / 9$ & $442,320,349$ & $17.88 \%$ & $8.28 \%$ \\
\hline 11 & $81 / 10$ & $536,571,926$ & $19.00 \%$ & $10.04 \%$ \\
\hline 12 & $81 / 11$ & $742,336,497$ & $20.16 \%$ & $13.89 \%$ \\
\hline 13 & $81 / 12$ & $822,436,935$ & $21.29 \%$ & $15.39 \%$ \\
\hline 14 & $82 / 1$ & $931,406,994$ & $22.45 \%$ & $17.43 \%$ \\
\hline 15 & $82 / 2$ & $987,661,260$ & $23.61 \%$ & $18.48 \%$ \\
\hline 16 & $82 / 3$ & $1,067,296,113$ & $24.66 \%$ & $19.97 \%$ \\
\hline 17 & $82 / 4$ & $1,162,841,603$ & $25.82 \%$ & $21.76 \%$ \\
\hline 18 & $82 / 5$ & $1,257,354,425$ & $26.95 \%$ & $23.53 \%$ \\
\hline 19 & $82 / 6$ & $1,320,772,152$ & $28.11 \%$ & $24.71 \%$ \\
\hline 20 & $82 / 7$ & $1,361,222,085$ & $29.24 \%$ & $25.47 \%$ \\
\hline 21 & $82 / 8$ & $1,447,318,620$ & $30.40 \%$ & $27.08 \%$ \\
\hline 22 & $82 / 9$ & $1,541,962,339$ & $31.56 \%$ & $28.85 \%$ \\
\hline 23 & $82 / 10$ & $1,629,858,563$ & $32.68 \%$ & $30.49 \%$ \\
\hline 24 & $82 / 11$ & $1,750,420,395$ & $33.85 \%$ & $32.75 \%$ \\
\hline 25 & $82 / 12$ & $1,898,633,071$ & $34.97 \%$ & $35.52 \%$ \\
\hline 26 & $83 / 1$ & $2,024,377,919$ & $36.13 \%$ & $37.88 \%$ \\
\hline 27 & $83 / 2$ & $2,103,877,262$ & $37.29 \%$ & $39.36 \%$ \\
\hline 28 & $83 / 3$ & $2,232,694,031$ & $38.34 \%$ & $41.77 \%$ \\
\hline 29 & $83 / 4$ & $2,519,705,280$ & $39.51 \%$ & $47.14 \%$ \\
\hline 30 & $83 / 5$ & $2,571,581,797$ & $40.63 \%$ & $48.11 \%$ \\
\hline 31 & $83 / 6$ & $2,629,026,261$ & $41.79 \%$ & $49.19 \%$ \\
\hline 32 & $83 / 7$ & $2,700,041,284$ & $42.92 \%$ & $50.52 \%$ \\
\hline 33 & $83 / 8$ & $2,747,993,528$ & $44.08 \%$ & $51.42 \%$ \\
\hline 34 & $83 / 9$ & $2,805,024,504$ & $45.24 \%$ & $52.48 \%$ \\
\hline 35 & $83 / 10$ & $2,935,567,858$ & $46.36 \%$ & $54.92 \%$ \\
\hline 36 & $83 / 11$ & $3,024,292,651$ & $47.53 \%$ & $56.58 \%$ \\
\hline 37 & $83 / 12$ & $3,107,403,233$ & $48.65 \%$ & $58.14 \%$ \\
\hline 38 & $84 / 1$ & $3,199,752,852$ & $49.81 \%$ & $59.87 \%$ \\
\hline 39 & $84 / 2$ & $3,258,272,603$ & $50.97 \%$ & $60.96 \%$ \\
\hline 40 & $84 / 3$ & $3,310,923,072$ & $52.02 \%$ & $61.95 \%$ \\
\hline 41 & $84 / 4$ & $3,362,164,118$ & $53.19 \%$ & $62.91 \%$ \\
\hline 42 & $84 / 5$ & $3,397,079,648$ & $54.31 \%$ & $63.56 \%$ \\
\hline 43 & $84 / 6$ & $3,460,527,952$ & $55.47 \%$ & $64.75 \%$ \\
\hline 44 & $84 / 7$ & $4,403,859,399$ & $56.60 \%$ & $82.40 \%$ \\
\hline 45 & $84 / 8$ & $4,446,190,555$ & $57.76 \%$ & $83.19 \%$ \\
\hline 46 & $84 / 9$ & $4,467,998,609$ & $58.92 \%$ & $83.60 \%$ \\
\hline 47 & $84 / 10$ & $4,491,507,050$ & $60.04 \%$ & $84.04 \%$ \\
\hline 48 & $84 / 11$ & $4,515,791,597$ & $61.21 \%$ & $84.49 \%$ \\
\hline 49 & $84 / 12$ & $4,533,959,483$ & $62.33 \%$ & $84.83 \%$ \\
\hline 50 & $85 / 1$ & $4,619,987,215$ & $63.49 \%$ & $86.44 \%$ \\
\hline 51 & $85 / 2$ & $4,657,660,695$ & $64.66 \%$ & $87.15 \%$ \\
\hline 52 & $85 / 3$ & $4,674,670,042$ & $65.74 \%$ & $87.46 \%$ \\
\hline 53 & $85 / 4$ & $4,692,964,462$ & $66.90 \%$ & $87.81 \%$ \\
\hline 54 & $85 / 5$ & $4,720,798,078$ & $68.03 \%$ & $88.33 \%$ \\
\hline 55 & $85 / 6$ & $4,761,901,206$ & $69.19 \%$ & $89.10 \%$ \\
\hline 56 & $85 / 7$ & $4,799,763,766$ & $70.31 \%$ & $89.80 \%$ \\
\hline 57 & $85 / 8$ & $4,831,040,196$ & $71.48 \%$ & $90.39 \%$ \\
\hline 58 & $85 / 9$ & $4,856,949,225$ & $72.64 \%$ & $90.87 \%$ \\
\hline 59 & $85 / 10$ & $4,893,195,670$ & $73.76 \%$ & $91.55 \%$ \\
\hline 60 & $85 / 11$ & $4,915,114,734$ & $74.93 \%$ & $91.96 \%$ \\
\hline 61 & $85 / 12$ & $4,931,559,544$ & $76.05 \%$ & $92.27 \%$ \\
\hline 62 & $86 / 1$ & $4,946,721,205$ & $77.21 \%$ & $92.55 \%$ \\
\hline 63 & $86 / 2$ & $4,980,138,304$ & $78.37 \%$ & $93.18 \%$ \\
\hline 64 & $86 / 3$ & $5,001,588,959$ & $79.42 \%$ & $93.58 \%$ \\
\hline 65 & $86 / 4$ & $5,034,731,706$ & $80.58 \%$ & $94.20 \%$ \\
\hline 66 & $86 / 5$ & $5,064,029,324$ & $81.71 \%$ & $94.75 \%$ \\
\hline 67 & $86 / 6$ & $5,080,106,114$ & $82.87 \%$ & $95.05 \%$ \\
\hline 68 & $86 / 7$ & $5,094,461,369$ & $84.00 \%$ & $95.32 \%$ \\
\hline 69 & $86 / 8$ & $5,104,146,881$ & $85.16 \%$ & $95.50 \%$ \\
\hline 70 & $86 / 9$ & $5,127,692,651$ & $86.32 \%$ & $95.94 \%$ \\
\hline 71 & $86 / 10$ & $5,130,617,000$ & $87.44 \%$ & $95.99 \%$ \\
\hline 72 & $86 / 11$ & $5,146,615,889$ & $88.61 \%$ & $96.29 \%$ \\
\hline 73 & $86 / 12$ & $5,167,787,282$ & $89.73 \%$ & $96.69 \%$ \\
\hline 74 & $87 / 1$ & $5,182,884,070$ & $90.89 \%$ & $96.97 \%$ \\
\hline 75 & $87 / 2$ & $5,195,927,731$ & $92.05 \%$ & $97.22 \%$ \\
\hline 76 & $87 / 3$ & $5,198,499,181$ & $93.10 \%$ & $97.26 \%$ \\
\hline 77 & $87 / 4$ & $5,229,133,485$ & $94.27 \%$ & $97.84 \%$ \\
\hline 78 & $87 / 5$ & $5,230,091,831$ & $95.39 \%$ & $97.86 \%$ \\
\hline 79 & $87 / 6$ & $5,233,925,940$ & $96.55 \%$ & $97.93 \%$ \\
\hline 80 & $87 / 7$ & $5,244,739,340$ & $97.68 \%$ & $98.13 \%$ \\
\hline 81 & $87 / 8$ & $5,261,551,354$ & $98.84 \%$ & $98.44 \%$ \\
\hline 82 & $87 / 9$ & $5,344,703,689$ & $100.00 \%$ & $100.00 \%$ \\
\hline
\end{tabular}

Table 1b. The observed data of the second metro bid

\begin{tabular}{|c|c|c|c|c|}
\hline $\begin{array}{l}\text { Valuation } \\
\text { times }\end{array}$ & $\begin{array}{l}\text { Valuation } \\
\text { month }\end{array}$ & $\begin{array}{l}\text { Accumulative } \\
\text { valuation total }\end{array}$ & $\mathrm{X} 2$ & $\mathrm{Y} 2$ \\
\hline $\begin{array}{l}\text { To begin } \\
\text { construction }\end{array}$ & $80 / 10$ & 0 & $0.00 \%$ & $0.00 \%$ \\
\hline 1 & $81 / 1$ & $1,156,545$ & $3.64 \%$ & $0.04 \%$ \\
\hline 2 & $81 / 2$ & $3,728,523$ & $4.87 \%$ & $0.14 \%$ \\
\hline 3 & $81 / 3$ & $7,053,156$ & $6.02 \%$ & $0.27 \%$ \\
\hline 4 & $81 / 4$ & $11,640,351$ & $7.24 \%$ & $0.45 \%$ \\
\hline 5 & $81 / 5$ & $14,165,122$ & $8.43 \%$ & $0.55 \%$ \\
\hline 6 & $81 / 6$ & $16,202,393$ & $9.66 \%$ & $0.63 \%$ \\
\hline 7 & $81 / 7$ & $19,325,192$ & $10.84 \%$ & $0.75 \%$ \\
\hline 8 & $81 / 8$ & $26,313,197$ & $12.07 \%$ & $1.02 \%$ \\
\hline 9 & $81 / 9$ & $33,874,345$ & $13.30 \%$ & $1.31 \%$ \\
\hline 10 & $81 / 10$ & $40,747,052$ & $14.48 \%$ & $1.58 \%$ \\
\hline 11 & $81 / 11$ & $45,312,485$ & $15.71 \%$ & $1.75 \%$ \\
\hline 12 & $82 / 1$ & $119,181,601$ & $18.12 \%$ & $4.61 \%$ \\
\hline 3 & $82 / 2$ & $120,818,877$ & $19.35 \%$ & $4.67 \%$ \\
\hline 14 & $82 / 3$ & $122,879,242$ & $20.46 \%$ & $4.75 \%$ \\
\hline 15 & $82 / 4$ & $133,756,647$ & $21.69 \%$ & $5.17 \%$ \\
\hline 16 & $82 / 5$ & $172,640,019$ & $22.87 \%$ & $6.68 \%$ \\
\hline 17 & $82 / 6$ & $210,552,786$ & $24.10 \%$ & $8.14 \%$ \\
\hline 18 & $82 / 7$ & $265,195,305$ & $25.29 \%$ & $10.25 \%$ \\
\hline 19 & $82 / 8$ & $294,185,718$ & $26.51 \%$ & $11.37 \%$ \\
\hline 20 & $82 / 9$ & $380,119,266$ & $27.74 \%$ & $14.70 \%$ \\
\hline 21 & $83 / 1$ & $459,243,280$ & $32.57 \%$ & $17.76 \%$ \\
\hline 22 & $83 / 2$ & $469,477,077$ & $33.80 \%$ & $18.15 \%$ \\
\hline 23 & $83 / 3$ & $487,586,298$ & $34.90 \%$ & $18.85 \%$ \\
\hline 24 & $83 / 4$ & $532,094,836$ & $36.13 \%$ & $20.57 \%$ \\
\hline 25 & $83 / 5$ & $644,916,728$ & $37.32 \%$ & $24.94 \%$ \\
\hline 26 & $83 / 6$ & $700,195,457$ & $38.54 \%$ & $27.07 \%$ \\
\hline 27 & $83 / 7$ & $738,333,537$ & $39.73 \%$ & $28.55 \%$ \\
\hline 28 & $83 / 8$ & $766,818,033$ & $40.96 \%$ & $29.65 \%$ \\
\hline 29 & $83 / 9$ & $792,154,251$ & $42.18 \%$ & $30.63 \%$ \\
\hline 30 & $83 / 10$ & $815,399,237$ & $43.37 \%$ & $31.53 \%$ \\
\hline 31 & $83 / 11$ & $846,562,020$ & $44.60 \%$ & $32.73 \%$ \\
\hline 32 & $83 / 12$ & $865,807,268$ & $45.79 \%$ & $33.48 \%$ \\
\hline 33 & $84 / 1$ & $1,380,442,059$ & $47.01 \%$ & $53.37 \%$ \\
\hline 34 & $84 / 2$ & $1,497,462,305$ & $48.24 \%$ & $57.90 \%$ \\
\hline 35 & $84 / 3$ & $1,525,953,929$ & $49.35 \%$ & $59.00 \%$ \\
\hline 36 & $84 / 4$ & $1,546,538,864$ & $50.57 \%$ & $59.80 \%$ \\
\hline 37 & $84 / 5$ & $1,570,538,279$ & $51.76 \%$ & $60.72 \%$ \\
\hline 38 & $84 / 6$ & $1,623,168,190$ & $52.99 \%$ & $62.76 \%$ \\
\hline 39 & $84 / 7$ & $1,644,431,305$ & $54.17 \%$ & $63.58 \%$ \\
\hline 40 & $84 / 8$ & $1,658,358,605$ & $55.40 \%$ & $64.12 \%$ \\
\hline 41 & $84 / 9$ & $1,684,287,284$ & $56.63 \%$ & $65.12 \%$ \\
\hline 42 & $84 / 10$ & $1,697,113,677$ & $57.82 \%$ & $65.62 \%$ \\
\hline 43 & $84 / 11$ & $1,711,041,422$ & $59.04 \%$ & $66.16 \%$ \\
\hline 44 & $84 / 12$ & $1,712,435,049$ & $60.23 \%$ & $66.21 \%$ \\
\hline 45 & $85 / 1$ & $1,719,528,978$ & $61.46 \%$ & $66.48 \%$ \\
\hline 46 & $85 / 2$ & $1,723,221,308$ & $62.68 \%$ & $66.63 \%$ \\
\hline 47 & $85 / 3$ & $1,727,736,424$ & $63.83 \%$ & $66.80 \%$ \\
\hline 48 & $85 / 4$ & $1,732,329,343$ & $65.06 \%$ & $66.98 \%$ \\
\hline 49 & $85 / 5$ & $1,737,137,366$ & $66.24 \%$ & $67.17 \%$ \\
\hline 50 & $85 / 6$ & $1,738,489,060$ & $67.47 \%$ & $67.22 \%$ \\
\hline 51 & $85 / 7$ & $1,749,472,473$ & $68.66 \%$ & $67.64 \%$ \\
\hline 52 & $85 / 8$ & $1,793,540,247$ & $69.89 \%$ & $69.35 \%$ \\
\hline 53 & $85 / 9$ & $1,827,170,765$ & $71.11 \%$ & $70.65 \%$ \\
\hline 54 & $85 / 10$ & $1,840,254,656$ & $72.30 \%$ & $71.15 \%$ \\
\hline 55 & $85 / 11$ & $1,850,614,305$ & $73.53 \%$ & $71.55 \%$ \\
\hline 56 & $85 / 12$ & $1,863,435,430$ & $74.71 \%$ & $72.05 \%$ \\
\hline 57 & $86 / 1$ & $2,131,536,620$ & $75.94 \%$ & $82.41 \%$ \\
\hline 58 & $86 / 2$ & $2,158,399,172$ & $77.17 \%$ & $83.45 \%$ \\
\hline 59 & $86 / 3$ & $2,187,280,073$ & $78.27 \%$ & $84.57 \%$ \\
\hline 60 & $86 / 4$ & $2,220,208,512$ & $79.50 \%$ & $85.84 \%$ \\
\hline 61 & $86 / 5$ & $2,250,661,259$ & $80.69 \%$ & $87.02 \%$ \\
\hline 62 & $86 / 6$ & $2,297,727,304$ & $81.92 \%$ & $88.84 \%$ \\
\hline 63 & $86 / 7$ & $2,310,868,650$ & $83.10 \%$ & $89.35 \%$ \\
\hline 64 & $86 / 8$ & $2,320,802,152$ & $84.33 \%$ & $89.73 \%$ \\
\hline 65 & $86 / 9$ & $2,343,720,959$ & $85.56 \%$ & $90.62 \%$ \\
\hline 66 & $86 / 11$ & $2,379,705,456$ & $87.97 \%$ & $92.01 \%$ \\
\hline 67 & $86 / 12$ & $2,430,838,711$ & $89.16 \%$ & $93.99 \%$ \\
\hline 68 & $87 / 1$ & $2,480,724,529$ & $90.38 \%$ & $95.92 \%$ \\
\hline 69 & $87 / 2$ & $2,498,827,432$ & $91.61 \%$ & $96.62 \%$ \\
\hline 70 & $87 / 3$ & $2,520,545,261$ & $92.72 \%$ & $97.46 \%$ \\
\hline 71 & $87 / 4$ & $2,532,296,991$ & $93.95 \%$ & $97.91 \%$ \\
\hline 72 & $87 / 5$ & $2,543,924,217$ & $95.13 \%$ & $98.36 \%$ \\
\hline 73 & $87 / 6$ & $2,550,673,441$ & $96.36 \%$ & $98.62 \%$ \\
\hline 74 & $87 / 7$ & $2,564,337,596$ & $97.55 \%$ & $99.15 \%$ \\
\hline 75 & $87 / 8$ & $2,571,281,827$ & $98.77 \%$ & $99.42 \%$ \\
\hline 76 & $87 / 9$ & $2,586,362,110$ & $100.00 \%$ & $100.00 \%$ \\
\hline
\end{tabular}


Table 1c. The observed data of the third metro bid

\begin{tabular}{|c|c|c|c|c|}
\hline $\begin{array}{l}\text { Valuation } \\
\text { times }\end{array}$ & $\begin{array}{c}\text { Valuation } \\
\text { month }\end{array}$ & $\begin{array}{l}\text { Accumulative } \\
\text { valuation total }\end{array}$ & $\mathrm{X} 3$ & Y3 \\
\hline $\begin{array}{l}\text { To begin } \\
\text { construction }\end{array}$ & $81 / 7$ & 0 & $0.00 \%$ & $0.00 \%$ \\
\hline 1 & $81 / 10$ & 770,150 & $3.29 \%$ & $0.04 \%$ \\
\hline 2 & $81 / 11$ & $3,660,218$ & $4.39 \%$ & $0.21 \%$ \\
\hline 3 & $81 / 12$ & $6,468,329$ & $5.46 \%$ & $0.36 \%$ \\
\hline 4 & $82 / 1$ & $8,744,528$ & $6.57 \%$ & $0.49 \%$ \\
\hline 5 & $82 / 2$ & $9,540,080$ & $7.68 \%$ & $0.54 \%$ \\
\hline 6 & $82 / 3$ & $10,466,539$ & $8.68 \%$ & $0.59 \%$ \\
\hline 7 & $82 / 4$ & $30,587,616$ & $9.79 \%$ & $1.73 \%$ \\
\hline 8 & $82 / 5$ & $57,283,475$ & $10.86 \%$ & $3.23 \%$ \\
\hline 9 & $82 / 6$ & $90,958,299$ & $11.96 \%$ & $5.13 \%$ \\
\hline 10 & $82 / 7$ & $128,780,447$ & $13.04 \%$ & $7.27 \%$ \\
\hline 11 & $82 / 8$ & $178,058,830$ & $14.14 \%$ & $10.05 \%$ \\
\hline 12 & $82 / 9$ & $233,101,158$ & $15.25 \%$ & $13.15 \%$ \\
\hline 13 & $82 / 10$ & $279,934,914$ & $16.32 \%$ & $15.79 \%$ \\
\hline 14 & $82 / 11$ & $315,939,427$ & $17.43 \%$ & $17.82 \%$ \\
\hline 15 & $82 / 12$ & $331,823,791$ & $18.50 \%$ & $18.72 \%$ \\
\hline 16 & $83 / 1$ & $384,135,189$ & $19.61 \%$ & $21.67 \%$ \\
\hline 17 & $83 / 2$ & $404,360,405$ & $20.71 \%$ & $22.81 \%$ \\
\hline 18 & $83 / 3$ & $420,393,448$ & $21.71 \%$ & $23.72 \%$ \\
\hline 19 & $83 / 4$ & $448,483,677$ & $22.82 \%$ & $25.30 \%$ \\
\hline 20 & $83 / 5$ & $471,861,908$ & $23.89 \%$ & $26.62 \%$ \\
\hline 21 & $83 / 6$ & $500,240,139$ & $25.00 \%$ & $28.22 \%$ \\
\hline 22 & $83 / 7$ & $516,704,749$ & $26.07 \%$ & $29.15 \%$ \\
\hline 23 & $83 / 8$ & $552,712,519$ & $27.18 \%$ & $31.18 \%$ \\
\hline 24 & $83 / 9$ & $616,268,555$ & $28.29 \%$ & $34.77 \%$ \\
\hline 25 & $83 / 10$ & $638,781,109$ & $29.36 \%$ & $36.04 \%$ \\
\hline 26 & $83 / 11$ & $670,837,936$ & $30.46 \%$ & $37.85 \%$ \\
\hline 27 & $83 / 12$ & $696,804,627$ & $31.54 \%$ & $39.31 \%$ \\
\hline 28 & $84 / 1$ & $747,886,459$ & $32.64 \%$ & $42.19 \%$ \\
\hline 29 & $84 / 2$ & $780,734,148$ & $33.75 \%$ & $44.05 \%$ \\
\hline 30 & $84 / 3$ & $811,864,699$ & $34.75 \%$ & $45.80 \%$ \\
\hline 31 & $84 / 4$ & $845,871,058$ & $35.86 \%$ & $47.72 \%$ \\
\hline 32 & $84 / 5$ & $867,506,050$ & $36.93 \%$ & $48.94 \%$ \\
\hline 33 & $84 / 6$ & $917,912,126$ & $38.04 \%$ & $51.79 \%$ \\
\hline 34 & $84 / 7$ & $952,643,465$ & $39.11 \%$ & $53.75 \%$ \\
\hline 35 & $84 / 8$ & $987,249,602$ & $40.21 \%$ & $55.70 \%$ \\
\hline 36 & $84 / 9$ & $1,006,968,362$ & $41.32 \%$ & $56.81 \%$ \\
\hline 37 & $84 / 10$ & $1,050,964,004$ & $42.39 \%$ & $59.29 \%$ \\
\hline 38 & $84 / 11$ & $1,068,425,625$ & $43.50 \%$ & $60.28 \%$ \\
\hline 39 & $84 / 12$ & $1,087,530,687$ & $44.57 \%$ & $61.36 \%$ \\
\hline 40 & $85 / 1$ & $1,120,856,728$ & $45.68 \%$ & $63.24 \%$ \\
\hline 41 & $85 / 2$ & $1,163,552,902$ & $46.79 \%$ & $65.65 \%$ \\
\hline 42 & $85 / 3$ & $1,205,617,726$ & $47.82 \%$ & $68.02 \%$ \\
\hline 43 & $85 / 4$ & $1,226,324,572$ & $48.93 \%$ & $69.19 \%$ \\
\hline 44 & $85 / 5$ & $1,236,342,045$ & $50.00 \%$ & $69.75 \%$ \\
\hline 45 & $85 / 6$ & $1,256,566,420$ & $51.11 \%$ & $70.89 \%$ \\
\hline 46 & $85 / 7$ & $1,277,850,992$ & $52.18 \%$ & $72.09 \%$ \\
\hline 47 & $85 / 8$ & $1,290,013,052$ & $53.29 \%$ & $72.78 \%$ \\
\hline 48 & $85 / 9$ & $1,295,975,943$ & $54.39 \%$ & $73.12 \%$ \\
\hline 49 & $85 / 10$ & $1,315,728,497$ & $55.46 \%$ & $74.23 \%$ \\
\hline 50 & $85 / 11$ & $1,330,760,753$ & $56.57 \%$ & $75.08 \%$ \\
\hline 51 & $85 / 12$ & $1,341,051,277$ & $57.64 \%$ & $75.66 \%$ \\
\hline 52 & $86 / 1$ & $1,352,369,641$ & $58.75 \%$ & $76.30 \%$ \\
\hline 53 & $86 / 2$ & $1,358,357,473$ & $59.86 \%$ & $76.64 \%$ \\
\hline 54 & $86 / 3$ & $1,362,707,210$ & $60.86 \%$ & $76.88 \%$ \\
\hline 55 & $86 / 4$ & $1,372,404,716$ & $61.96 \%$ & $77.43 \%$ \\
\hline 56 & $86 / 5$ & $1,378,980,471$ & $63.04 \%$ & $77.80 \%$ \\
\hline 57 & $86 / 6$ & $1,395,853,072$ & $64.14 \%$ & $78.75 \%$ \\
\hline 58 & $86 / 7$ & $1,412,018,013$ & $65.21 \%$ & $79.66 \%$ \\
\hline 59 & $86 / 8$ & $1,418,774,059$ & $66.32 \%$ & $80.04 \%$ \\
\hline 60 & $86 / 9$ & $1,428,797,828$ & $67.43 \%$ & $80.61 \%$ \\
\hline 61 & $86 / 10$ & $1,432,738,639$ & $68.50 \%$ & $80.83 \%$ \\
\hline 62 & $86 / 11$ & $1,438,702,459$ & $69.61 \%$ & $81.17 \%$ \\
\hline 63 & $86 / 12$ & $1,444,497,877$ & $70.68 \%$ & $81.50 \%$ \\
\hline 64 & $87 / 1$ & $1,446,168,246$ & $71.79 \%$ & $81.59 \%$ \\
\hline 65 & $87 / 2$ & $1,449,373,445$ & $72.89 \%$ & $81.77 \%$ \\
\hline 66 & $87 / 3$ & $1,463,579,040$ & $73.89 \%$ & $82.57 \%$ \\
\hline 67 & $87 / 4$ & $1,468,590,317$ & $75.00 \%$ & $82.85 \%$ \\
\hline 68 & $87 / 5$ & $1,500,100,500$ & $76.07 \%$ & $84.63 \%$ \\
\hline 69 & $87 / 6$ & $1,515,408,037$ & $77.18 \%$ & $85.50 \%$ \\
\hline 70 & $87 / 7$ & $1,521,528,896$ & $78.25 \%$ & $85.84 \%$ \\
\hline 71 & $87 / 8$ & $1,527,702,009$ & $79.36 \%$ & $86.19 \%$ \\
\hline 72 & $87 / 9$ & $1,540,236,294$ & $80.46 \%$ & $86.90 \%$ \\
\hline 73 & $87 / 10$ & $1,556,406,732$ & $81.54 \%$ & $87.81 \%$ \\
\hline 74 & $87 / 11$ & $1,566,018,131$ & $82.64 \%$ & $88.35 \%$ \\
\hline 75 & $87 / 12$ & $1,587,020,792$ & $83.71 \%$ & $89.54 \%$ \\
\hline 76 & $88 / 1$ & $1,598,887,531$ & $84.82 \%$ & $90.21 \%$ \\
\hline 77 & $88 / 2$ & $1,603,173,509$ & $85.93 \%$ & $90.45 \%$ \\
\hline 78 & $88 / 3$ & $1,618,830,901$ & $86.93 \%$ & $91.33 \%$ \\
\hline 79 & $88 / 4$ & $1,628,781,452$ & $88.04 \%$ & $91.89 \%$ \\
\hline 80 & $88 / 5$ & $1,654,153,614$ & $89.11 \%$ & $93.32 \%$ \\
\hline 81 & $88 / 6$ & $1,723,126,058$ & $90.21 \%$ & $97.22 \%$ \\
\hline 82 & $88 / 8$ & $1,724,596,923$ & $92.39 \%$ & $97.30 \%$ \\
\hline 83 & $88 / 10$ & $1,756,578,360$ & $94.57 \%$ & $99.10 \%$ \\
\hline 84 & $89 / 1$ & $1,762,491,332$ & $97.86 \%$ & $99.44 \%$ \\
\hline 85 & $89 / 3$ & $1,772,482,708$ & $100.00 \%$ & $100.00 \%$ \\
\hline
\end{tabular}

Table 1d. The observed data of the 4th metro bid

\begin{tabular}{|c|c|c|c|c|}
\hline $\begin{array}{l}\text { Valuation } \\
\text { times }\end{array}$ & $\begin{array}{c}\text { Valuation } \\
\text { month }\end{array}$ & $\begin{array}{l}\text { Accumulative } \\
\text { valuation total }\end{array}$ & $\mathrm{X} 4$ & Y4 \\
\hline $\begin{array}{c}\text { To begin } \\
\text { construction }\end{array}$ & $81 / 1$ & 0 & $0.00 \%$ & $0.00 \%$ \\
\hline 1 & $81 / 4$ & $9,717,588$ & $3.25 \%$ & $0.25 \%$ \\
\hline 2 & $81 / 5$ & $14,452,656$ & $4.32 \%$ & $0.38 \%$ \\
\hline 3 & $81 / 6$ & $20,292,030$ & $5.43 \%$ & $0.53 \%$ \\
\hline 4 & $81 / 7$ & $33,230,588$ & $6.50 \%$ & $0.87 \%$ \\
\hline 5 & $81 / 8$ & $36,803,660$ & $7.61 \%$ & $0.96 \%$ \\
\hline 6 & $81 / 9$ & $39,030,540$ & $8.71 \%$ & $1.02 \%$ \\
\hline 7 & $81 / 10$ & $58,392,258$ & $9.79 \%$ & $1.52 \%$ \\
\hline 8 & $81 / 11$ & $61,404,357$ & $10.89 \%$ & $1.60 \%$ \\
\hline 9 & $81 / 12$ & $63,700,019$ & $11.96 \%$ & $1.66 \%$ \\
\hline 10 & $82 / 1$ & $76,507,432$ & $13.07 \%$ & $2.00 \%$ \\
\hline 11 & $82 / 2$ & $80,702,452$ & $14.18 \%$ & $2.11 \%$ \\
\hline 12 & $82 / 3$ & $124,158,031$ & $15.18 \%$ & $3.24 \%$ \\
\hline 13 & $82 / 4$ & $141,275,103$ & $16.29 \%$ & $3.69 \%$ \\
\hline 14 & $82 / 5$ & $158,256,672$ & $17.36 \%$ & $4.13 \%$ \\
\hline 15 & $82 / 6$ & $214,734,109$ & $18.46 \%$ & $5.61 \%$ \\
\hline 16 & $82 / 7$ & $241,802,878$ & $19.54 \%$ & $6.31 \%$ \\
\hline 17 & $82 / 8$ & $277,317,105$ & $20.64 \%$ & $7.24 \%$ \\
\hline 18 & $82 / 9$ & $301,646,427$ & $21.75 \%$ & $7.88 \%$ \\
\hline 19 & $82 / 10$ & $343,260,841$ & $22.82 \%$ & $8.96 \%$ \\
\hline 20 & $82 / 11$ & $411,486,177$ & $23.93 \%$ & $10.75 \%$ \\
\hline 21 & $82 / 12$ & $458,279,034$ & $25.00 \%$ & $11.97 \%$ \\
\hline 22 & $83 / 1$ & $503,693,842$ & $26.11 \%$ & $13.15 \%$ \\
\hline 23 & $83 / 2$ & $573,992,217$ & $27.21 \%$ & $14.99 \%$ \\
\hline 24 & $83 / 3$ & $599,929,009$ & $28.21 \%$ & $15.67 \%$ \\
\hline 25 & $83 / 4$ & $631,090,609$ & $29.32 \%$ & $16.48 \%$ \\
\hline 26 & $83 / 5$ & $703,922,093$ & $30.39 \%$ & $18.38 \%$ \\
\hline 27 & $83 / 6$ & $742,878,532$ & $31.50 \%$ & $19.40 \%$ \\
\hline 28 & $83 / 7$ & $780,853,820$ & $32.57 \%$ & $20.39 \%$ \\
\hline 29 & $83 / 8$ & $817,428,123$ & $33.68 \%$ & $21.35 \%$ \\
\hline 30 & $83 / 9$ & $840,862,895$ & $34.79 \%$ & $21.96 \%$ \\
\hline 31 & $83 / 10$ & $898,536,513$ & $35.86 \%$ & $23.47 \%$ \\
\hline 32 & $83 / 11$ & $942,509,760$ & $36.96 \%$ & $24.61 \%$ \\
\hline 33 & $83 / 12$ & $1,044,902,352$ & $38.04 \%$ & $27.29 \%$ \\
\hline 34 & $84 / 1$ & $1,158,880,592$ & $39.14 \%$ & $30.27 \%$ \\
\hline 35 & $84 / 2$ & $1,184,351,532$ & $40.25 \%$ & $30.93 \%$ \\
\hline 36 & $84 / 3$ & $1,218,684,512$ & $41.25 \%$ & $31.83 \%$ \\
\hline 37 & $84 / 4$ & $1,245,689,628$ & $42.36 \%$ & $32.53 \%$ \\
\hline 38 & $84 / 5$ & $1,271,673,941$ & $43.43 \%$ & $33.21 \%$ \\
\hline 39 & $84 / 6$ & $1,294,753,195$ & $44.54 \%$ & $33.81 \%$ \\
\hline 40 & $84 / 7$ & $1,518,131,791$ & $45.61 \%$ & $39.65 \%$ \\
\hline 41 & $84 / 8$ & $1,714,198,207$ & $46.71 \%$ & $44.77 \%$ \\
\hline 42 & $84 / 9$ & $1,783,621,592$ & $47.82 \%$ & $46.58 \%$ \\
\hline 43 & $84 / 10$ & $1,845,495,018$ & $48.89 \%$ & $48.20 \%$ \\
\hline 44 & $84 / 11$ & $1,924,914,284$ & $50.00 \%$ & $50.27 \%$ \\
\hline 45 & $84 / 12$ & $2,059,183,278$ & $51.07 \%$ & $53.78 \%$ \\
\hline 46 & $85 / 1$ & $2,160,490,338$ & $52.18 \%$ & $56.42 \%$ \\
\hline 47 & $85 / 2$ & $2,340,027,081$ & $53.29 \%$ & $61.11 \%$ \\
\hline 48 & $85 / 3$ & $2,446,181,017$ & $54.32 \%$ & $63.89 \%$ \\
\hline 49 & $85 / 4$ & $2,478,213,303$ & $55.43 \%$ & $64.72 \%$ \\
\hline 50 & $85 / 5$ & $2,489,499,064$ & $56.50 \%$ & $65.02 \%$ \\
\hline 51 & $85 / 6$ & $2,491,949,283$ & $57.61 \%$ & $65.08 \%$ \\
\hline 52 & $85 / 7$ & $2,560,875,043$ & $58.68 \%$ & $66.88 \%$ \\
\hline 53 & $85 / 8$ & $2,609,003,087$ & $59.79 \%$ & $68.14 \%$ \\
\hline 54 & $85 / 9$ & $2,666,691,388$ & $60.89 \%$ & $69.64 \%$ \\
\hline 55 & $85 / 10$ & $2,684,622,178$ & $61.96 \%$ & $70.11 \%$ \\
\hline 56 & $85 / 11$ & $2,700,243,113$ & $63.07 \%$ & $70.52 \%$ \\
\hline 57 & $85 / 12$ & $2,751,078,967$ & $64.14 \%$ & $71.85 \%$ \\
\hline 58 & $86 / 1$ & $2,817,796,220$ & $65.25 \%$ & $73.59 \%$ \\
\hline 59 & $86 / 2$ & $2,836,173,261$ & $66.36 \%$ & $74.07 \%$ \\
\hline 60 & $86 / 3$ & $2,859,819,153$ & $67.36 \%$ & $74.69 \%$ \\
\hline 61 & $86 / 4$ & $2,868,127,716$ & $68.46 \%$ & $74.90 \%$ \\
\hline 62 & $86 / 5$ & $2,877,930,823$ & $69.54 \%$ & $75.16 \%$ \\
\hline 63 & $86 / 6$ & $2,894,348,761$ & $70.64 \%$ & $75.59 \%$ \\
\hline 64 & $86 / 7$ & $2,914,840,708$ & $71.71 \%$ & $76.12 \%$ \\
\hline 65 & $86 / 8$ & $2,938,418,345$ & $72.82 \%$ & $76.74 \%$ \\
\hline 66 & $86 / 9$ & $2,948,724,619$ & $73.93 \%$ & $77.01 \%$ \\
\hline 67 & $86 / 10$ & $2,963,782,956$ & $75.00 \%$ & $77.40 \%$ \\
\hline 68 & $86 / 11$ & $2,991,148,829$ & $76.11 \%$ & $78.12 \%$ \\
\hline 69 & $86 / 12$ & $3,003,984,537$ & $77.18 \%$ & $78.45 \%$ \\
\hline 70 & $87 / 1$ & $3,064,820,124$ & $78.29 \%$ & $80.04 \%$ \\
\hline 71 & $87 / 2$ & $3,136,645,130$ & $79.39 \%$ & $81.92 \%$ \\
\hline 72 & $87 / 3$ & $3,222,054,828$ & $80.39 \%$ & $84.15 \%$ \\
\hline 73 & $87 / 4$ & $3,266,282,921$ & $81.50 \%$ & $85.30 \%$ \\
\hline 74 & $87 / 5$ & $3,283,787,821$ & $82.57 \%$ & $85.76 \%$ \\
\hline 75 & $87 / 6$ & $3,317,376,633$ & $83.68 \%$ & $86.64 \%$ \\
\hline 76 & $87 / 7$ & $3,349,582,153$ & $84.75 \%$ & $87.48 \%$ \\
\hline 77 & $87 / 8$ & $3,373,093,925$ & $85.86 \%$ & $88.09 \%$ \\
\hline 78 & $87 / 9$ & $3,393,205,437$ & $86.96 \%$ & $88.62 \%$ \\
\hline 79 & $87 / 10$ & $3,417,732,181$ & $88.04 \%$ & $89.26 \%$ \\
\hline 80 & $87 / 11$ & $3,440,252,746$ & $89.14 \%$ & $89.85 \%$ \\
\hline 81 & $87 / 12$ & $3,476,282,372$ & $90.21 \%$ & $90.79 \%$ \\
\hline 82 & $88 / 1$ & $3,493,605,664$ & $91.32 \%$ & $91.24 \%$ \\
\hline 83 & $88 / 2$ & $3,512,035,658$ & $92.43 \%$ & $91.72 \%$ \\
\hline 84 & $88 / 3$ & $3,535,261,109$ & $93.43 \%$ & $92.33 \%$ \\
\hline 85 & $88 / 4$ & $3,585,095,640$ & $94.54 \%$ & $93.63 \%$ \\
\hline 86 & $88 / 5$ & $3,586,666,772$ & $95.61 \%$ & $93.67 \%$ \\
\hline 87 & $88 / 6$ & $3,591,453,546$ & $96.71 \%$ & $93.80 \%$ \\
\hline 88 & $88 / 7$ & $3,733,326,575$ & $97.79 \%$ & $97.50 \%$ \\
\hline 89 & $88 / 8$ & $3,735,184,113$ & $98.89 \%$ & $97.55 \%$ \\
\hline 90 & $88 / 9$ & $3,829,030,861$ & $100.00 \%$ & $100.00 \%$ \\
\hline
\end{tabular}


Table 1e. The observed data of the 5th metro bid

\begin{tabular}{|c|c|c|c|c|}
\hline $\begin{array}{c}\text { Valuation } \\
\text { times }\end{array}$ & $\begin{array}{c}\text { Valuation } \\
\text { month }\end{array}$ & $\begin{array}{l}\text { Accumulative } \\
\text { valuation total }\end{array}$ & $\mathrm{X} 5$ & Y5 \\
\hline $\begin{array}{l}\text { To begin } \\
\text { construction }\end{array}$ & $81 / 1$ & 0 & $0.00 \%$ & $0.00 \%$ \\
\hline 1 & $81 / 4$ & $3,157,984$ & $3.52 \%$ & $0.17 \%$ \\
\hline 2 & $81 / 7$ & $5,611,645$ & $7.03 \%$ & $0.30 \%$ \\
\hline 3 & $81 / 8$ & $9,425,952$ & $8.23 \%$ & $0.51 \%$ \\
\hline 4 & $81 / 9$ & $10,299,491$ & $9.43 \%$ & $0.56 \%$ \\
\hline 5 & $81 / 11$ & $12,764,490$ & $11.79 \%$ & $0.69 \%$ \\
\hline 7 & $82 / 1$ & $54,334,131$ & $14.14 \%$ & $2.94 \%$ \\
\hline 8 & $82 / 2$ & $75,551,887$ & $15.34 \%$ & $4.08 \%$ \\
\hline 9 & $82 / 3$ & $86,630,800$ & $16.42 \%$ & $4.68 \%$ \\
\hline 10 & $82 / 4$ & $103,048,314$ & $17.62 \%$ & $5.57 \%$ \\
\hline 11 & $82 / 5$ & $139,609,556$ & $18.78 \%$ & $7.55 \%$ \\
\hline 12 & $82 / 6$ & $173,056,321$ & $19.98 \%$ & $9.35 \%$ \\
\hline 13 & $82 / 7$ & $204,721,783$ & $21.14 \%$ & $11.07 \%$ \\
\hline 14 & $82 / 8$ & $222,398,269$ & $22.33 \%$ & $12.02 \%$ \\
\hline 15 & $82 / 9$ & $224,404,386$ & $23.53 \%$ & $12.13 \%$ \\
\hline 16 & $82 / 11$ & $234,237,185$ & $25.89 \%$ & $12.66 \%$ \\
\hline 17 & $82 / 12$ & $256,533,899$ & $27.05 \%$ & $13.87 \%$ \\
\hline 18 & $83 / 1$ & $297,716,885$ & $28.25 \%$ & $16.09 \%$ \\
\hline 19 & $83 / 2$ & $305,459,380$ & $29.44 \%$ & $16.51 \%$ \\
\hline 20 & $83 / 3$ & $335,755,912$ & $30.53 \%$ & $18.15 \%$ \\
\hline 21 & $83 / 4$ & $340,022,082$ & $31.72 \%$ & $18.38 \%$ \\
\hline 22 & $83 / 5$ & $351,026,511$ & $32.88 \%$ & $18.97 \%$ \\
\hline 23 & $83 / 6$ & $398,589,232$ & $34.08 \%$ & $21.54 \%$ \\
\hline 24 & $83 / 7$ & $424,014,518$ & $35.24 \%$ & $22.92 \%$ \\
\hline 25 & $83 / 8$ & $440,038,388$ & $36.44 \%$ & $23.79 \%$ \\
\hline 26 & $83 / 9$ & $451,674,151$ & $37.64 \%$ & $24.41 \%$ \\
\hline 27 & $83 / 11$ & $475,557,405$ & $39.99 \%$ & $25.71 \%$ \\
\hline 28 & $83 / 12$ & $482,752,644$ & $41.15 \%$ & $26.09 \%$ \\
\hline 29 & $84 / 1$ & $550,811,248$ & $42.35 \%$ & $29.77 \%$ \\
\hline 30 & $84 / 2$ & $657,849,322$ & $43.55 \%$ & $35.56 \%$ \\
\hline 31 & $84 / 3$ & $814,932,958$ & $44.63 \%$ & $44.05 \%$ \\
\hline 32 & $84 / 4$ & $925,735,504$ & $45.83 \%$ & $50.04 \%$ \\
\hline 33 & $84 / 5$ & $1,014,368,688$ & $46.99 \%$ & $54.83 \%$ \\
\hline 34 & $84 / 6$ & $1,021,366,008$ & $48.18 \%$ & $55.21 \%$ \\
\hline 35 & $84 / 7$ & $1,022,875,745$ & $49.34 \%$ & $55.29 \%$ \\
\hline 36 & $84 / 8$ & $1,023,432,791$ & $50.54 \%$ & $55.32 \%$ \\
\hline 37 & $84 / 9$ & $1,023,915,614$ & $51.74 \%$ & $55.35 \%$ \\
\hline 38 & $84 / 11$ & $1,024,398,433$ & $54.10 \%$ & $55.37 \%$ \\
\hline 39 & $84 / 12$ & $1,030,354,689$ & $55.26 \%$ & $55.69 \%$ \\
\hline 40 & $85 / 1$ & $1,035,104,573$ & $56.45 \%$ & $55.95 \%$ \\
\hline 41 & $85 / 2$ & $1,035,587,394$ & $57.65 \%$ & $55.98 \%$ \\
\hline 42 & $85 / 3$ & $1,036,070,213$ & $58.77 \%$ & $56.00 \%$ \\
\hline 43 & $85 / 4$ & $1,110,419,745$ & $59.97 \%$ & $60.02 \%$ \\
\hline 44 & $85 / 5$ & $1,220,897,645$ & $61.13 \%$ & $65.99 \%$ \\
\hline 45 & $85 / 6$ & $1,262,989,056$ & $62.33 \%$ & $68.27 \%$ \\
\hline 46 & $85 / 7$ & $1,332,751,092$ & $63.49 \%$ & $72.04 \%$ \\
\hline 47 & $85 / 8$ & $1,333,237,716$ & $64.68 \%$ & $72.07 \%$ \\
\hline 48 & $85 / 9$ & $1,333,773,802$ & $65.88 \%$ & $72.09 \%$ \\
\hline 49 & $85 / 11$ & $1,334,264,148$ & $68.24 \%$ & $72.12 \%$ \\
\hline 50 & $85 / 12$ & $1,343,207,704$ & $69.40 \%$ & $72.60 \%$ \\
\hline 51 & $86 / 1$ & $1,387,727,110$ & $70.60 \%$ & $75.01 \%$ \\
\hline 52 & $86 / 2$ & $1,540,850,336$ & $71.79 \%$ & $83.29 \%$ \\
\hline 53 & $86 / 3$ & $1,630,875,831$ & $72.87 \%$ & $88.15 \%$ \\
\hline 54 & $86 / 4$ & $1,631,046,356$ & $74.07 \%$ & $88.16 \%$ \\
\hline 55 & $86 / 5$ & $1,664,333,982$ & $75.23 \%$ & $89.96 \%$ \\
\hline 56 & $86 / 6$ & $1,666,382,775$ & $76.43 \%$ & $90.07 \%$ \\
\hline 57 & $86 / 7$ & $1,666,539,178$ & $77.59 \%$ & $90.08 \%$ \\
\hline 58 & $86 / 8$ & $1,691,517,333$ & $78.79 \%$ & $91.43 \%$ \\
\hline 59 & $86 / 9$ & $1,694,658,162$ & $79.98 \%$ & $91.60 \%$ \\
\hline 60 & $86 / 11$ & $1,705,681,899$ & $82.34 \%$ & $92.20 \%$ \\
\hline 61 & $86 / 12$ & $1,727,987,625$ & $83.50 \%$ & $93.40 \%$ \\
\hline 62 & $87 / 1$ & $1,747,674,733$ & $84.70 \%$ & $94.47 \%$ \\
\hline 63 & $87 / 2$ & $1,753,386,399$ & $85.90 \%$ & $94.78 \%$ \\
\hline 64 & $87 / 3$ & $1,764,776,359$ & $86.98 \%$ & $95.39 \%$ \\
\hline 65 & $87 / 4$ & $1,772,226,875$ & $88.18 \%$ & $95.79 \%$ \\
\hline 66 & $87 / 5$ & $1,815,968,977$ & $89.34 \%$ & $98.16 \%$ \\
\hline 67 & $87 / 6$ & $1,828,265,501$ & $90.53 \%$ & $98.82 \%$ \\
\hline 68 & $87 / 7$ & $1,835,757,811$ & $91.69 \%$ & $99.23 \%$ \\
\hline 69 & $87 / 8$ & $1,838,287,114$ & $92.89 \%$ & $99.37 \%$ \\
\hline 70 & $87 / 9$ & $1,839,318,252$ & $94.09 \%$ & $99.42 \%$ \\
\hline 71 & $87 / 11$ & $1,846,237,199$ & $96.45 \%$ & $99.79 \%$ \\
\hline 72 & $88 / 2$ & $1,850,032,940$ & $100.00 \%$ & $100.00 \%$ \\
\hline
\end{tabular}

Table 1f. The observed data of the 6th metro bid

\begin{tabular}{|c|c|c|c|c|}
\hline $\begin{array}{l}\text { Valuation } \\
\text { times }\end{array}$ & $\begin{array}{l}\text { Valuation } \\
\text { month }\end{array}$ & $\begin{array}{l}\text { Accumulative } \\
\text { valuation total }\end{array}$ & X6 & Y6 \\
\hline $\begin{array}{c}\text { To begin } \\
\text { construction }\end{array}$ & $84 / 9$ & 0 & $0.00 \%$ & $0.00 \%$ \\
\hline 1 & $84 / 12$ & $1,196,429$ & $5.75 \%$ & $0.11 \%$ \\
\hline 2 & $85 / 1$ & $7,484,820$ & $7.71 \%$ & $0.66 \%$ \\
\hline 3 & $85 / 2$ & $9,108,509$ & $9.67 \%$ & $0.81 \%$ \\
\hline 4 & $85 / 3$ & $11,400,844$ & $11.50 \%$ & $1.01 \%$ \\
\hline 5 & $85 / 4$ & $12,655,783$ & $13.46 \%$ & $1.12 \%$ \\
\hline 6 & $85 / 5$ & $40,841,449$ & $15.35 \%$ & $3.62 \%$ \\
\hline 7 & $85 / 6$ & $52,346,329$ & $17.31 \%$ & $4.63 \%$ \\
\hline 8 & $85 / 8$ & $60,797,648$ & $21.16 \%$ & $5.38 \%$ \\
\hline 9 & $85 / 10$ & $60,995,847$ & $25.02 \%$ & $5.40 \%$ \\
\hline 10 & $85 / 11$ & $62,916,342$ & $26.97 \%$ & $5.57 \%$ \\
\hline 11 & $85 / 12$ & $67,254,060$ & $28.87 \%$ & $5.95 \%$ \\
\hline 12 & $86 / 1$ & $100,206,292$ & $30.83 \%$ & $8.87 \%$ \\
\hline 13 & $86 / 3$ & $102,784,371$ & $34.55 \%$ & $9.10 \%$ \\
\hline 14 & $86 / 5$ & $103,468,121$ & $38.41 \%$ & $9.16 \%$ \\
\hline 15 & $86 / 6$ & $114,992,782$ & $40.37 \%$ & $10.18 \%$ \\
\hline 16 & $86 / 7$ & $122,744,984$ & $42.26 \%$ & $10.87 \%$ \\
\hline 17 & $86 / 8$ & $143,160,179$ & $44.22 \%$ & $12.67 \%$ \\
\hline 18 & $86 / 9$ & $190,468,065$ & $46.18 \%$ & $16.86 \%$ \\
\hline 19 & $86 / 10$ & $239,013,770$ & $48.07 \%$ & $21.16 \%$ \\
\hline 20 & $86 / 11$ & $294,623,573$ & $50.03 \%$ & $26.08 \%$ \\
\hline 21 & $86 / 12$ & $412,771,998$ & $51.93 \%$ & $36.54 \%$ \\
\hline 22 & $87 / 1$ & $500,498,449$ & $53.89 \%$ & $44.31 \%$ \\
\hline 23 & $87 / 3$ & $513,633,770$ & $57.61 \%$ & $45.47 \%$ \\
\hline 24 & $87 / 4$ & $534,698,236$ & $59.57 \%$ & $47.34 \%$ \\
\hline 25 & $87 / 5$ & $590,543,842$ & $61.47 \%$ & $52.28 \%$ \\
\hline 26 & $87 / 6$ & $602,157,035$ & $63.42 \%$ & $53.31 \%$ \\
\hline 27 & $87 / 7$ & $618,978,284$ & $65.32 \%$ & $54.80 \%$ \\
\hline 28 & $87 / 8$ & $646,144,725$ & $67.28 \%$ & $57.20 \%$ \\
\hline 29 & $87 / 9$ & $685,602,225$ & $69.24 \%$ & $60.69 \%$ \\
\hline 30 & $87 / 10$ & $736,566,872$ & $71.13 \%$ & $65.21 \%$ \\
\hline 31 & $87 / 11$ & $765,215,105$ & $73.09 \%$ & $67.74 \%$ \\
\hline 32 & $87 / 12$ & $850,864,754$ & $74.98 \%$ & $75.33 \%$ \\
\hline 33 & $88 / 1$ & $874,446,506$ & $76.94 \%$ & $77.41 \%$ \\
\hline 34 & $88 / 2$ & $924,641,322$ & $78.90 \%$ & $81.86 \%$ \\
\hline 35 & $88 / 3$ & $1,012,565,332$ & $80.67 \%$ & $89.64 \%$ \\
\hline 36 & $88 / 4$ & $1,026,516,972$ & $82.63 \%$ & $90.88 \%$ \\
\hline 37 & $89 / 1$ & $1,129,589,930$ & $100.00 \%$ & $100.00 \%$ \\
\hline
\end{tabular}

discrete and delayed situation. The process of the former $30 \%$ duration of the project is a bit slow. In addition, the first evaluation time of total duration is $4.5 \%$ when a contractor proposes. It implies that we must notice the delayed situation of cash flow and maintain the liquidity of cash portion to some degree to ensure the project can be finished smoothly and successfully.

\section{ACKNOWLEDGEMENT}

This paper is supported in part by the National Science Council of Taiwan, R.O.C. under projects No.NSC 91-2626-E-238-003.

\section{REFERENCES}

1. Chatterjee, S. and Price, B., Regression Analysis by Example, John Wiley and Sons, Inc., New York (1991).

2. Diamond, P., "Fuzzy Least Squares," Inform. Sci., Vol. 46, pp. 141-157 (1988).

3. Kaka, A.P., "Contractors' Financial Budgeting Using Computer Simulation," Construc. Manag. Econ., Vol. 12, pp. 113-124 (1994).

4. Klir, G.J. and Yuan, B., Fuzzy Sets and Fuzzy Logic Theory and Applications, Prentic-Hall, Englewood 
Cliffs, NJ (1995).

5. Miskawi, Z., "An $S$-curve Equation for Project Control," Construc. Manag. Econ., Vol. 7, pp. 115-124 (1989).

6. Ruoning, X., "A Linear Regression Model in Fuzzy Environment," Adv. Model. Simul., Vol. 27, pp. 31-40 (1991).

7. Ruoning, X., "S-curve Regression Model in Fuzzy Environment," Fuzzy Sets Syst., Vol. 90, pp. 317-326 (1997).

8. Takagi, T. and Sugeno, M., "Fuzzy Identification of Systems and Its Applications to Modeling and Control," IEEE Trans. Syst., Man, Cybernetics, Vol. 15, pp.
116-132 (1985).

9. Tanaka, H., Uejima, S., and Asai, K. "Linear Regression Analysis with Fuzzy Model," IEEE Trans. Syst., Man, Cybernetics, Vol. 12, pp. 903-907 (1982).

10. Wang, H.O., Tanaka, K., and Griffin, M.F., “An Approach to Fuzzy Control of Nonlinear Systems: Stability and Design Issues," IEEE Trans. Fuzzy Syst., Vol. 4, pp. 14-23 (1996).

11. Wang, W.J. and Chiu, C.H., "Entropy Variation on The Fuzzy Numbers with Arithmetic Operations," Fuzzy Sets Syst., Vol. 103, No. 3, pp. 443-456 (1999).

12. Zadeh, L.A., "Fuzzy Sets," Inform. Control, Vol. 8, pp. 338-353 (1965). 